\title{
SEIZING EFFECT IN THE ROLLER BURNISHING PROCESS
}

\author{
W. GAMBIN \\ Institute of Fundamental Technological Research, Polish Academy of Sciences \\ Świętokrzyska 21, 00-049 Warsaw, Poland
}

Micrographs of metal surface layers after the roller burnishing process show that while about $90 \%$ of surface layer thickness is displaced forwards of a roller motion, a thin contact zone undergoes a backward displacement. This seizing effect is caused by a softening of the contact zone due to overheating of the material, during high speed rolling, or due to the plastic strain cumulation in the layer after multiple rolling repetition. The effect is responsible for a peeling and cracking of surface layers during the burnishing process. The plastic analysis of the process taking into account the above effect is presented in the paper. The proposed slip-line field and corresponding velocity field are modification of the simplified solution for the rolling of the rigid-plastic half-space given by Collins.

PACS numbers: $68.10 . \mathrm{Cr}, 68.35 . \mathrm{Gy}$

\section{Introduction}

The roller burnishing process is one of mechanical treatments of metal surface layers. It may be considered as a particular case of the rolling contact problem [1-3], when the force-driven rigid cylinder is rolled without friction over a metal element. According to the Collins solution [1] the rolled surface undergoes a cumulative horizontal displacement in the direction of the roller motion. This fact is confirmed by many metallographical observations. However, in some cases [4-5], a thin direct contact zone undergoes a backwards displacements (Fig. 1).

The above seizing effect is connected with a decrease in the material hardness and softening of the material structure in the direct contact zone. The softening is caused by: a cumulation of the plastic strain (the highest in this zone) after successive passing of the roller; and/or an increase in temperature in this zone due to a high speed of the burnishing process. The seizing effect leads to a peeling and cracking of surface layers and-makes a serious problem in the engineering practice. It takes place for higher loads when the softening of the material due to rolling exceeds a certain critical value. The description of this effect and determination of the bounds for the corresponding loads are the aim of the paper. 


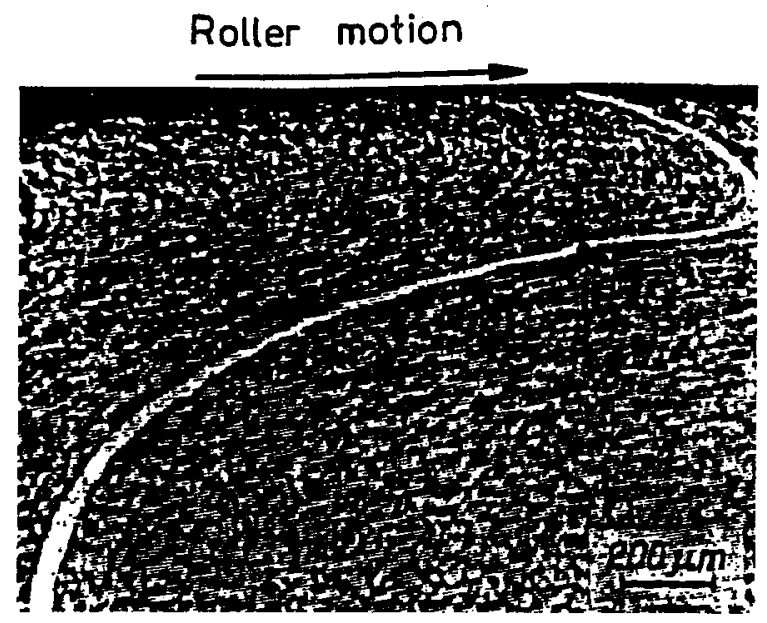

Fig. 1. Micrograph of metal surface layer after many cycles of roller burnishing.

\section{Classical solution for frictionless rolling}

Let us reconsider the classical solution [1] for the case of frictionless loading. A rigid cylinder is rolling on a rigid-perfectly plastic half-space with a constant speed $V$. There is no slipping on the contact surface. The vertical load $W$ and the horizontal load $F$ applied to the centre of the roller are shown in Fig. 2a. The material of the half-space is described by the yield shear stress $k$ only, and the cylinder radius $R$ is the only scale-defining parameter. Then, one can introduce the dimensionless load variables: $w=W / k R$ and $f=F / k R$. Because the arc of the contact between the cylinder and the surface is small as compared to the radius $R$, the arc may be approximated by the corresponding chord.

According to this solution the slip-line field is composed of two regions ADF. and ABC, in which $\alpha$ and $\beta$ slip-lines are straight, and a centred fan ACD with
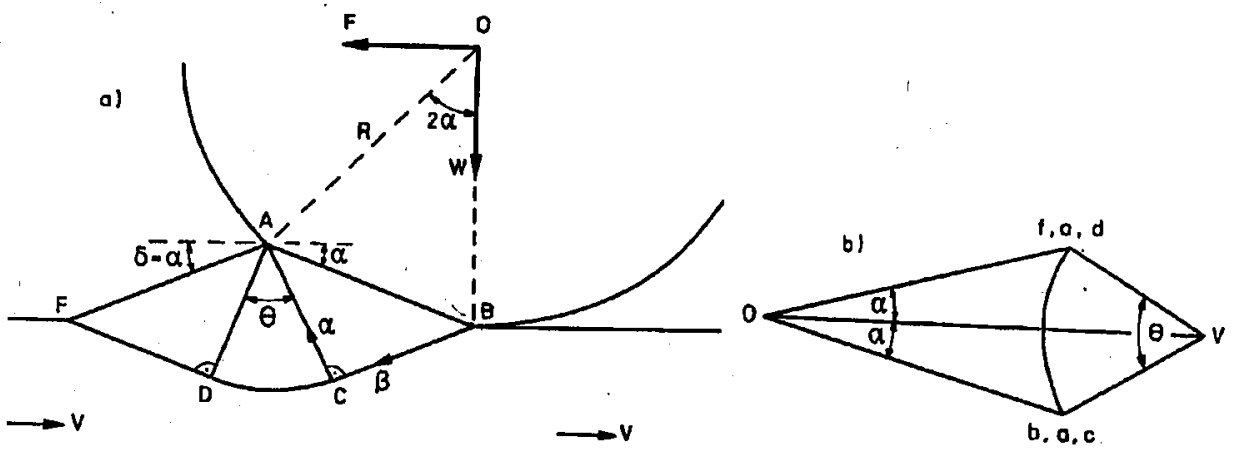

Fig. 2. Classical solution for frictionless rolling; (a) slip-line field, (b) hodograph. 
straight $\alpha$-lines. Let us denote the inclination of the chord AB by $\alpha$, and the inclination of the free surface FA by $\delta$. To satisfy the incompressibility condition at the plane strain state, $\alpha$ must be equal to $\delta$. The vertical angle $\theta$ in the centred fan ADC is determined by the rule: $\theta=\pi / 2-2 \alpha$. The standard application of Hencky's theorem [6] enables one to find the vertical and horizontal components of the load acting on the cylinder. For small angles $\alpha$, they are given by the rules

$$
w=2(1+\theta) \alpha \text { and } f=2(2+\pi) \alpha^{2}
$$

and the coefficient of the rolling friction $\mu_{\mathrm{R}} \equiv f / w$ is proportional to the vertical load

$$
\mu_{\mathrm{R}} \equiv w / 2(2+\pi) .
$$

According to the hodograph shown in Fig. 2b, the regions ABC and ADF move rigidly regarding the rigid part of the half-space in the direction $\mathrm{BC}$ and $\mathrm{DF}$, respectively. In the centred fan $A C D$, the velocity is constant along $\beta$-lines and a discontinuity of the velocity appears along FDCB. Because there is no slipping on the chord $A B$, the velocity $U$ of the rigid material in $A B C$ is the same like the peripheral speed of the cylinder. It yields from the hodograph that this speed is smaller than the advance speed of the material below the line FDCB. Then

$$
U=\frac{\cos (\alpha+\pi / 4)}{\cos (\pi / 4)} V \leq V
$$

It means that the surface layer is displaced always forwards of the roller motion and the seizing effect cannot be described within the classical model.

\section{Solution describing the seizing effect}

After too many cycles of rolling, a cumulation of plastic strains initiates microcracks in a thin contact layer. On the other hand, a high speed of the burnishing process causes an increase in temperature in this layer. Both effects lead to the softening of the material in the considered zone. Concluding, we assume that behind a certain front line dividing the contact surface, within a thin layer, a lower yield shear stress $k_{0} \leq k$ appears. A thickness of this layer is small as compared to the depth of the whole plastified layer, but it is large enough to contain an additional plastic zone that may appear due to the softening.

Let us modify the model shown in Fig. 2a penetrating slightly the cylinder into the half-space as it is shown in Fig. 3a. The penetration is determined by the angle $\varepsilon$, which describes the inclination of the tangent to the cylinder at the point $G$. The arc of the contact is fixed by the chord AB with the inclination $\alpha$ to the rolled surface. This arc will be approximated by two chords AC and CB with the inclination $\alpha+\varepsilon / 2$ and $\varepsilon / 2$, respectively. The inclination of the free surface $\mathrm{AF}$ in front of the cylinder is determined by the angle $\delta$. The transient contact surface BG is assumed to be not loaded and is regarded as a free surface. The angles: $\alpha$, $\delta$ and $\varepsilon$, are non-negative, and the angle $2 \alpha$ is not smaller than $\varepsilon$. For high loads applied to the roller (the case of the hardening burnishing process), the point $\mathrm{C}$ is close to $\mathrm{B}$ and the angle $\varepsilon$ is much smaller than $2 \alpha$.

The slip-line field is composed of two independent parts: $A_{F D} D_{2} C$ and $\mathrm{BCE}_{1} \mathrm{E}_{2} \mathrm{G}$. Each of them is constructed in the same way as in the classical model 

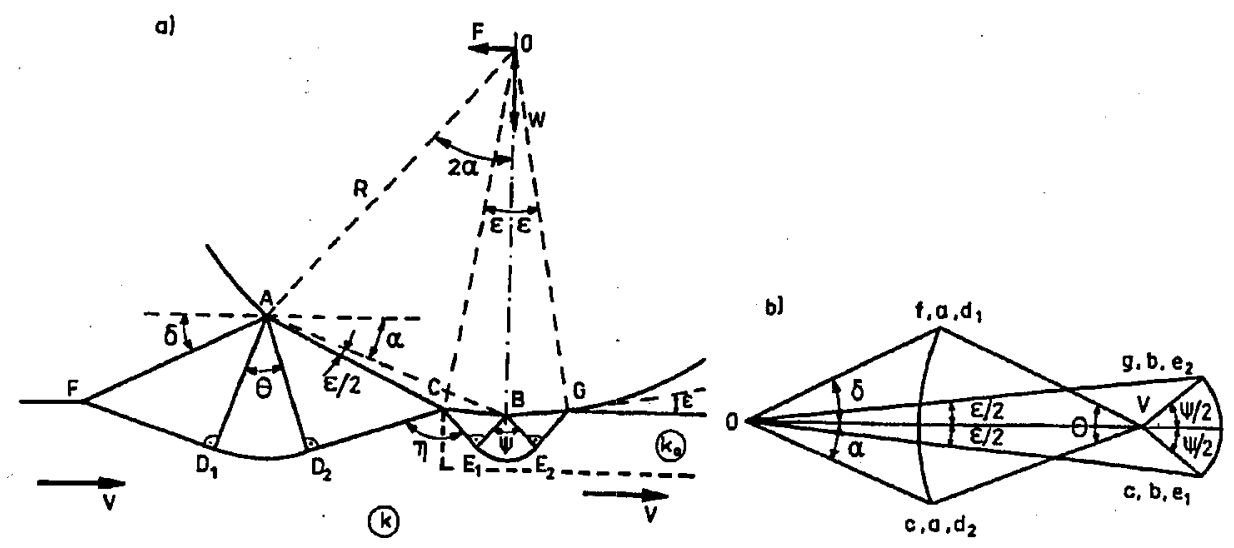

Fig. 3. Proposed solution describing the seizing effect; (a) slip-line field, (b) hodograph.

and it contains two triangles and one centred fan. The angles of the fans $A D_{1} D_{2}$ and $\mathrm{BE}_{1} \mathrm{E}_{2}$ are $\theta$ and $\psi$, respectively. The thickness of the first plastic zone is $\sqrt{2} R \sin \alpha$, and of the second one is $\sqrt{2} R \sin (\varepsilon / 2)$, where $R$ is the roller radius. For the hardening burnishing process the thickness of the second part of the slip-line field is much smaller than the thickness of the first one.

The problem is completely described once the angle $\alpha$ is specified. Then, we need four relations to determine the angles $\delta, \varepsilon, \theta$ and $\psi$ as functions of the angle $\alpha$. Moreover, the solution should depend on the ratio $k / k_{0}$, which describes the softening of the material during passing the roller. The analysis of the problem leads to the following relations:

$$
\begin{aligned}
& \delta=\alpha+\varepsilon / 2 \quad \alpha+\varepsilon / 2+\delta+\theta=\pi / 2, \\
& \psi-\varepsilon=\pi / 2, \quad k_{0}(1+2 \psi)=k(1+2 \theta) .
\end{aligned}
$$

The above relations fully determine the angles $\delta, \varepsilon, \theta$ and $\psi$ as functions of the angle $\alpha$.

Basing on Hencky's theorem [6], one can calculate the vertical and horizontal component of the force acting on the cylinder. Performing the calculation, we obtain the expressions (1). It means that the introduced modification of Collins' solution does not change the rolling resistance described by the rule (2).

Examining the hodograph shown in Fig. $3 \mathrm{~b}$, we see that the plastic region in front of the cylinder moves forwards of the roller motion like in Collins' solution. Now, let us consider the motion of the remaining plastic zone. Because the peripheral speed of the cylinder $U_{0}$ is the same as that of the rigid material in $\mathrm{BE}_{2} \mathrm{G}$, from the hodograph diagram it follows that

$$
U_{0}=\frac{\sin (\varepsilon+\pi / 4)}{\sin (\pi / 4)} V \geq V
$$

Then, the second plastic zone moves backwards concerning the roller motion. Putting together the displacements of both regions, we obtain the situation shown in Fig. 1. 


\section{Bounds for the new solution}

Looking at Fig. 3a, one can state that the following inequality must be satisfied: $2 \alpha \geq \varepsilon$. The relations (4) enable one to express $\varepsilon$ by $\alpha$. Then, the mentioned inequality give a certain restriction imposed on the angle $\alpha$. According to the rule (1), it gives a restriction on the vertical load $W$

$$
(1+\pi)(2+\pi) k R \frac{1-\kappa}{5+\kappa} \leq W \leq \frac{1}{2}(1+\pi)(2+\pi) k R(1-\kappa),
$$

where $k$ is the nominal yield shear stress, $k_{0}$ - the yield shear stress of the softened zone, $R$ is the roller radius and $\kappa=k_{0} / k$.

Thus, for a prescribed load $W$, the seizing effect occurs when the softening of the material is sufficiently high. One can decrease the softening by the reduction of the speed of the burnishing process or by the reduction of the number of rolling cycles. These manipulations diminish also the size of the plastic zone under the bottom of the roller. If the softening is small enough, the seizing effect disappears.

\section{Conclusions}

During the roller burnishing process with a high speed or after many cycles of rolling, a softening of a thin surface layer of the rolled material may appear. The proposed plastic analysis of the rolling process takes this fact into account. According to this analysis the relations between the applied loads and the length of the contact zone are the same as those given by the classical model [1]. However, the shape of slip-line field and the corresponding kinematics are different. Besides the bulk plastic region in front of the rolling cylinder, a small plastic zone of the softened material appears under the bottom of the roller. The softened material is seized by the roller and displaced backwards concerning the roller motion. The large plastic strains of the opposite signs cause a cumulation of microcracks in the material. Eventually, it leads to a peeling and cracking of metal surface layers in the burnished machine elements. If we know the softening of the material during the roller burnishing process, we can avoid the undesirable effects keeping the vertical load applied to the roller out of the bounds prescribed by the inequalities.

\section{Acknowledgment}

The paper is supported by the Committee for Scientific Research (grant PB 3 P402 016 07).

\section{References}

[1] I.F. Collins, Int. J. Mech. Sci. 14, 1 (1972).

[2] I.F. Collins, J. Mec. Appl. 2, 431 (1978).

[3] H. Petryk, Int. J. Mech. Sci. 25, 265 (1983).

[4] G. Pahlitzsch, P. Krohn, Werkstattstechnik 1 (1966).

[5] W. Przybylski, Burnishing Technology, WNT, Warszawa 1987 (in Polish).

[6] R. Hill, The Mathematical Theory of Plasticity, Clarendon Press, Oxford 1950. 